\title{
Desarrollo de una muleta sensorizada para medir la inclinación y el peso descargado
}

\author{
Iñigo Sesar, Aitziber Mancisidor, Asier Brull, Asier Zubizarreta, Itziar Cabanes \\ \{inigo.sesar, aitziber.mancisidor, asier.brull, asier.zubizarreta, itziar.cabanes\}@ehu.eus \\ Departamento de Ingeniería de Sistemas y Automática. \\ Escuela de Ingeniería de Bilbao (UPV/EHU)
}

\begin{abstract}
Resumen
Las escalas clínicas tradicionales utilizadas para el diagnóstico del estado funcional de pacientes con enfermedades neurológicas son subjetivas y poseen baja resolución. Sin embargo, se pueden incluir sensores de fuerza y movimiento en dispositivos pasivos de rehabilitación para realizar un diagnóstico objetivo y cuantitativo. En este trabajo, se presenta el diseño de una muleta instrumentada que incluye un sensor de fuerza, un inclinómetro y una IMU compuesta por un acelerómetro y un giróscopo de tres ejes. Además, se muestran los valores de la fuerza de apoyo e inclinación para un caso de estudio. La inclinación estimada por el inclinómetro y la IMU se valida con una cámara.
\end{abstract}

Palabras clave: Muleta, bastón, sensores vestibles, monitorización, análisis de la marcha.

\section{INTRODUCCIÓN}

Enfermedades neurológicas como la esclerosis múltiple y el ictus, o lesiones de la médula espinal son causas frecuentes por las que millones de personas pierden parte de su movilidad. Cada año, se diagnostican más de 15 millones de casos de ictus, siendo una de las principales causas de discapacidad [1]. Además, unas 850 personas por cada millón de habitantes sufren una lesión de la médula espinal [2]. Sin embargo, las escalas clínicas que se utilizan más frecuentemente para el diagnóstico de estas enfermedades o lesiones son subjetivas y proporcionan una resolución limitada, lo que dificulta determinar con precisión cuál es el estado funcional del paciente y cuál es la terapia de rehabilitación más adecuada $[3,4]$.

Para evaluar la calidad de movimiento y la mejora del estado funcional de los pacientes gracias a las terapias de rehabilitación, se pueden emplear indicadores cinemáticos, basados en las medidas de diversos sensores [3]. Estos indicadores pueden monitorizar el movimiento del paciente durante un tiempo prolongado y proporcionar una medida más precisa que las escalas clínicas tradicionales [4].
Dentro de la monitorización para el diagnóstico y rehabilitación de enfermedades neurológicas, cabe destacar la importancia de la monitorización de la marcha, ya que los pacientes que sufren una discapacidad en alguna extremidad inferior suelen tener una autonomía reducida, una peor salud física y una menor calidad de vida [2].

Con el fin de realizar una monitorización objetiva, se han planteado diversas soluciones. Por ejemplo, los sistemas de captura de movimiento más modernos son capaces de calcular la posición y orientación instantánea de cada parte del cuerpo, con un error inferior a $1 \mathrm{~mm}$ [5]. Estos sistemas están compuestos por una serie de cámaras situadas en distintos puntos de una sala y son capaces de medir la posición instantánea de unos marcadores reflectantes. Sin embargo, el espacio en el que estos sistemas pueden tomar las medidas suele ser reducido, generalmente son sistemas caros y requieren mucho tiempo para su calibración y para procesar los datos registrados [6].

Otra alternativa es emplear sensores vestibles o llevables (wearable sensors) para monitorizar la marcha. Los sensores vestibles son dispositivos electrónicos que se pueden atar al cuerpo o integrar en una prenda de ropa y que proporcionan información sobre el movimiento de los usuarios [7]. Además de plantearse como una solución no-invasiva, tienen la ventaja de que las medidas de los sensores pueden ser capturadas durante un tiempo prolongado en una gran variedad de entornos [7]. Además, los sensores vestibles pueden ahorrar costes, contribuyen a la mejora de la calidad de vida y requieren poco tiempo para la calibración y el procesamiento de datos $[6,8]$. Estos dispositivos pueden incluir sensores como acelerómetros, giróscopos, magnetómetros, barómetros, sensores de fuerza y receptores de GPS [6].

Los sensores vestibles también pueden ser integrados en dispositivos de ayuda para la marcha[8]. En concreto, varios trabajos han investigado el uso de pequeños sensores integrados en una muleta, la cual es un dispositivo pasivo utilizado en la rehabilitación de la marcha $[2,9,10,11,12,16,21]$. 
Estas soluciones presentan importantes ventajas frente al uso de sensores vestibles atados al cuerpo. Por ejemplo, los sensores se pueden unir rígidamente a la muleta, de forma que la orientación y posición del sensor respecto a la muleta es prácticamente constante. Esto no sucede si se coloca un sensor en el cuerpo, porque el movimiento del cuerpo provoca aceleraciones que pueden producir un desplazamiento relativo entre el sensor y los tejidos humanos [13]. Además, en los sistemas comercializados basados en sensores vestibles atados al cuerpo, el tiempo de ajuste y puesta en marcha varía entre 5 y 10 minutos con cada usuario $[14,15]$, mientras que, en los prototipos de muletas sensorizadas, este tiempo es de unos pocos segundos [10, 21].

Adicionalmente, una muleta instrumentada se puede emplear en muchas aplicaciones. Por ejemplo, se puede usar para prevenir caídas y detectar determinadas situaciones durante la marcha [12]. También se han utilizado muletas sensorizadas para medir el peso descargado sobre la muleta, de forma que el paciente pueda controlar la fuerza ejercida sobre la pierna afectada $[9,10,21]$. En [11], se utiliza un bastón instrumentado para estimar la intención de movimiento y poder controlar un exoesqueleto. También se pueden usar estos dispositivos para el entrenamiento de la marcha con muletas, monitorizando parámetros como la inclinación de la muleta o la desviación del centro de gravedad [17], ya que un uso inadecuado de la muleta puede provocar lesiones [9]. Incluso se pueden utilizar para la clasificación de diferentes ejercicios o actividades como subir y bajar escaleras, caminar en llano o quedarse de pie [9, 21]. Por último, también se pueden emplear para estimar la cantidad de energía consumida [18], la cual es un parámetro relacionado directamente con la fatiga de los pacientes [19].

En resumen, una muleta instrumentada puede aportar información que puede ser empleada para estimar unos indicadores que sirvan para realizar un diagnóstico objetivo y cuantitativo de la marcha en pacientes que sufren enfermedades neurológicas. Además de ser una solución ligera, flexible y no invasiva que puede tener varias aplicaciones, permite monitorizar los parámetros durante un tiempo prolongado y en numerosos entornos. Por ello, en este trabajo se presenta un sistema que se puede unir a la punta de diferentes muletas para estimar la inclinación de la muleta y la fuerza aplicada sobre ella. A diferencia de las publicaciones encontradas en la bibliografía, este trabajo presenta los resultados de utilizar un sistema de visión para validar la estimación de la inclinación durante la marcha, obtenida a partir de las medidas de los sensores integrados en la muleta.

El resto del artículo se organiza de la siguiente manera: en el apartado 2, se detalla el prototipo de la muleta; en el apartado 3, se plantea un caso de estudio para validar el diseño realizado, y por último, se detallan las ideas más relevantes.

\section{DESCRIPCIÓN DEL PROTOTIPO}

\subsection{Descripción general y especificaciones}

Como se muestra en la Figura 1, el sistema desarrollado está compuesto por una contera de aluminio que integra una serie de sensores, una batería, un dispositivo de adquisición de datos y una manguera de cables que conecta la contera con el dispositivo de adquisición. Esta manguera cuenta con un conector para poder desconectar la contera fácilmente. La batería y el dispositivo de adquisición van atados al cuerpo mediante un cinturón de neopreno.

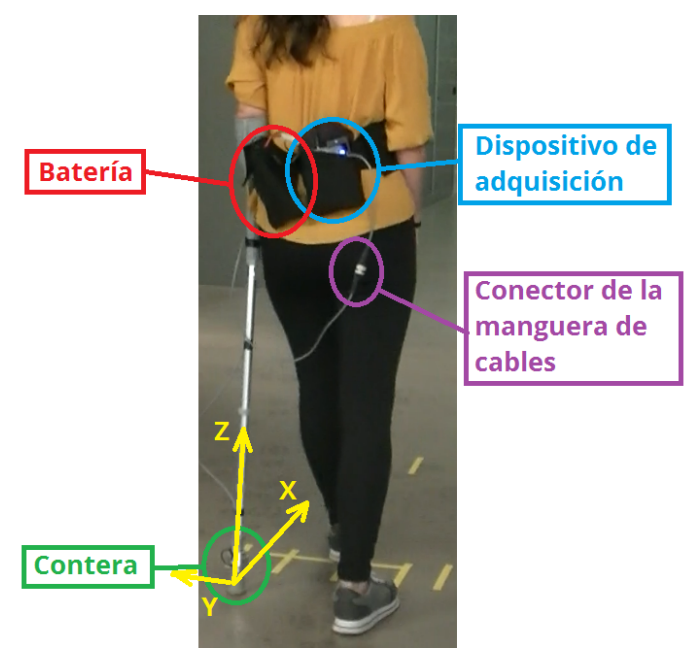

Figura 1: Perspectiva del sistema completo

Como se observa en la Figura 1, los ejes de la muleta se han elegido de forma que el eje $\mathrm{Z}$ coincida con el eje longitudinal de la muleta, el eje $\mathrm{X}$ con el eje del puño donde se apoya la mano y el eje $\mathrm{Y}$ acorde a los otros dos ejes, para formar un sistema dextrógiro. Por tanto, la inclinación anteroposterior será la rotación alrededor del eje Y de la muleta y la inclinación lateromedial será la rotación alrededor del eje X.

Debido a las limitaciones de los pacientes con enfermedades neurológicas, la batería y el dispositivo de adquisición elegidos no van unidos a la muleta, ya que esta pesaría demasiado. Adicionalmente, el prototipo se ha diseñado para cumplir con las siguientes especificaciones: posibilidad de ajuste a diferentes alturas, reducido tiempo de ajuste a 
cada paciente, facilidad de uso, sencillez, comodidad, ergonomía, portabilidad, bajo consumo energético y bajo coste. Además, en el diseño se ha considerado que el prototipo no obstaculice a los usuarios, que tenga la suficiente rigidez como para soportar al menos $120 \mathrm{Kg}$ y que la contera pueda ajustarse a diferentes tacos y diámetros de tubos.

En cuanto a las variables monitorizadas, se registran la fuerza aplicada en el eje longitudinal de la muleta, la aceleración y velocidad angular en los tres ejes, la inclinación anteroposterior, la inclinación lateromedial y determinados tiempos durante las tareas realizadas en los ensayos, como por ejemplo, el tiempo que el usuario necesita para recorrer una distancia de diez metros. A partir de esta información, se pueden obtener indicadores como la velocidad media, la cadencia media, la media del porcentaje del peso corporal descargado sobre la muleta (PBW), el número de pasos y la inclinación de la muleta en cada pico de fuerza.

\subsection{Sensores y sistema de adquisición de datos}

La fuerza aplicada en el eje $\mathrm{Z}$ de la muleta se mide con un sensor de fuerza C9C de HBM, el cual está diseñado para medir fuerzas de compresión estáticas y dinámicas de hasta 1200 N (máxima fuerza operativa). El sensor de fuerza pesa $65 \mathrm{~g}$ y presenta una no-linealidad inferior al 0,2\% FS (Full Scale). Dado que la tensión diferencial a la salida del sensor de fuerza es de solo $5 \mathrm{mV}$ con carga nominal, se ha empleado un amplificador de bajo consumo INA118, comercializado por Texas Instruments.

La posición angular alrededor de los ejes X e $\mathrm{Y}$ se mide mediante un inclinómetro de dos ejes SCA100T-D02, fabricado por Murata. Este sensor es capaz de medir en el rango de $\pm 90^{\circ}$ con una no-linealidad inferior a $1,3 \%$ FS y una sensibilidad entre ejes de $4 \%$ FS.

Para medir las aceleraciones y velocidades angulares en los tres ejes, se ha empleado la placa MPU IMU Click de MikroElektronika, la cual incluye el integrado MPU-6000 de InvenSense, compuesto por un acelerómetro triaxial, un giróscopo triaxial y una unidad de procesamiento de movimiento (MPU).

Las medidas de los sensores son adquiridas mediante una myRIO de National Instruments, la cual es un dispositivo reconfigurable de adquisición de señales tanto analógicas como digitales. La alimentación de la myRIO se obtiene de una batería recargable de $\mathrm{NiMH}$, fabricada por Ansmann, con una capacidad de 3000 mAh y una tensión de 7,2 V. El conjunto de la myRIO y batería pesa $553 \mathrm{~g}$ y consume menos de $14 \mathrm{~W}$, por lo que el sistema cumple las especificaciones de peso reducido y bajo consumo.

\subsection{Diseño mecánico}

Para integrar los sensores en la punta de la muleta, se diseñaron y fabricaron las piezas mostradas en la Figura 2(a). Estas piezas fabricadas en aluminio 6061 se unen mediante tornillos de acero inoxidable de cabeza allen para formar el ensamblaje mostrado en la figura 2(b). Se puede observar que el sensor de fuerza y la placa electrónica van montadas a ambos lados de un disco de aluminio de 7 $\mathrm{mm}$ de espesor, lo que garantiza suficiente rigidez. Este disco se une a una carcasa que protege el sensor de fuerza.

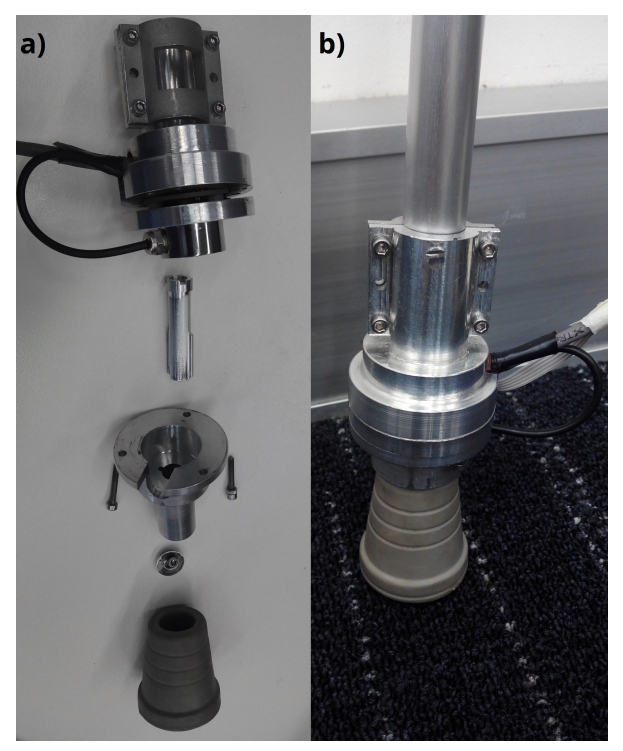

Figura 2: (a) Despiece de las partes mecanizadas. (b) Ensamblaje montado en la muleta.

\subsection{Diseño del software}

El programa para la adquisición de datos se ha desarrollado en LabView y se carga en la memoria de la myRIO. Este programa cuenta con un panel frontal o interfaz de usuario y un diagrama de bloques. La Figura 3 muestra una parte de la interfaz desarrollada en LabView. En el panel frontal, el usuario puede ver la duración de cada iteración del bucle de tiempo real, las medidas de todos los sensores y algunas otras variables, como el estado actual y una variable que muestra si se ha creado un archivo para registrar las medidas. Además, incluye un panel de control, donde el usuario puede cambiar el nombre del archivo, comenzar y terminar la captura de datos, resetear la myRIO, y registrar los tiempos en los que se aprieta un botón.

El diagrama de bloques del programa de LabView 


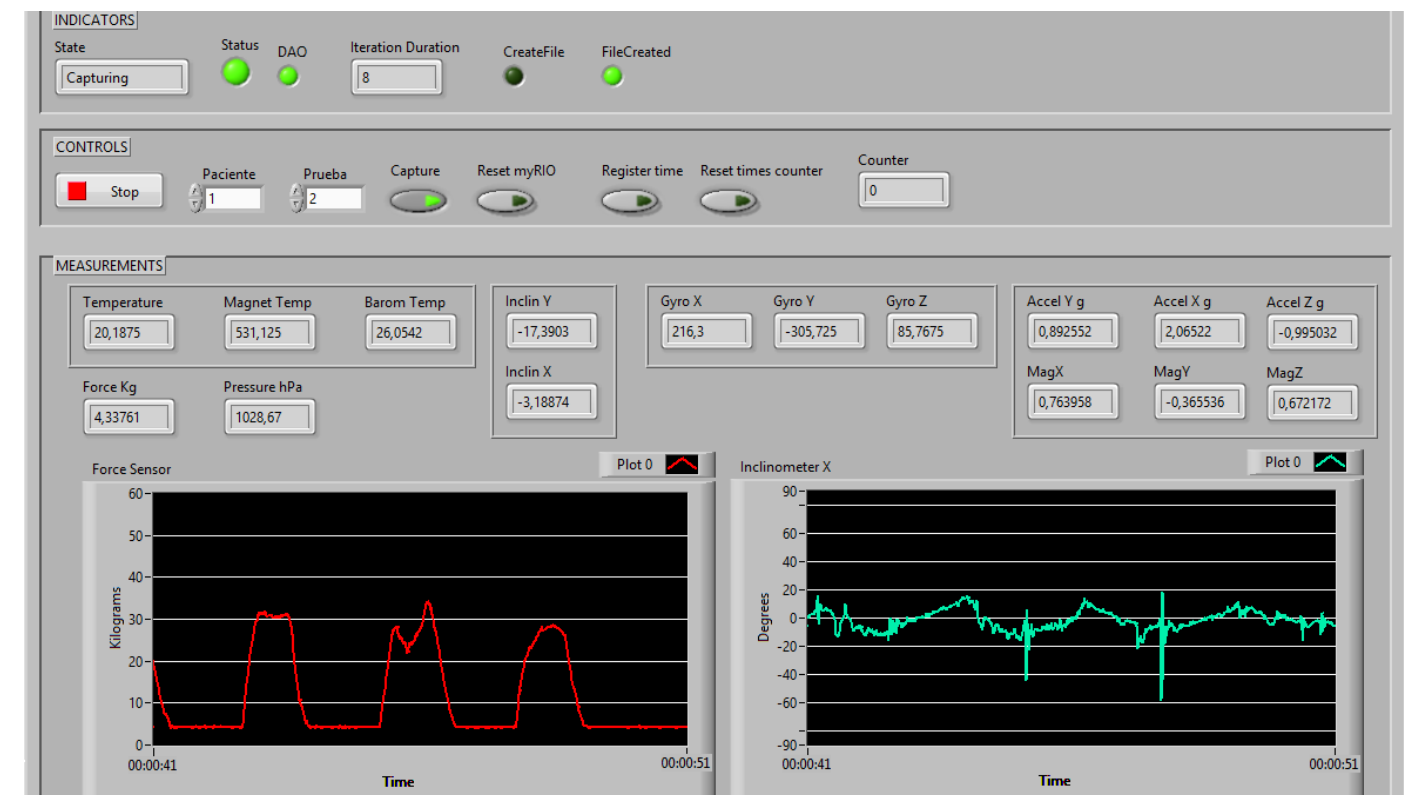

Figura 3: Parte del panel frontal del programa desarrollado en LabView.

está compuesto por un bucle de control y un bucle temporizado de tiempo real. El bucle de control se ejecuta cada 100 ms y cuenta con una máquina de estados que consta de 3 estados: inicialización, espera y captura.

- En el estado de incialización, se resetea la myRIO y se pasa al estado de espera. De forma concurrente, se configuran los sensores de la MPU-6000, según los parámetros elegidos por el usuario. Los registros $1 \mathrm{~B}$ y $1 \mathrm{C}$ se ponen a cero, para que el rango de medida del giróscopo sea $\pm 250^{\circ} / \mathrm{s}$ y el del acelerómetro \pm 2 g. También se ponen a cero los bits del registro $6 \mathrm{~B}$, para utilizar el oscilador interno de $8 \mathrm{MHz}$. Antes de entrar en el bucle de tiempo real, se espera medio segundo para que la IMU tenga suficiente tiempo para inicializar los registros y aplicar la configuración seleccionada. Como el bit AD0 se conectó a tierra, la dirección binaria del integrado para el protocolo I2C es 1101001.

- En el estado de espera, el programa espera a que el usuario apriete el botón de captura o el botón de reset en el panel frontal. Si se aprieta el botón de captura, se pasa al estado de captura, donde se crea un archivo con el nombre elegido por el usuario en la carpeta correspondiente de la memoria de la myRIO y se activa el indicador de archivo creado. La extensión del archivo creado es LVM y es un fichero de texto separado por tabulador que puede ser leído por cualquier hoja de cálculo.

- En el estado de captura, la myRIO registra las medidas de los sensores mediante el bucle de tiempo real hasta que el usuario apriete de nuevo el botón de captura, en cuyo caso volverá al estado de espera. El programa cerrará el archivo y finalizará cuando el usuario haga clic en el botón Stop del panel frontal, apriete el botón de la myRIO o se detecte algún error tras finalizar la captura.

El bucle de tiempo real se ejecuta con una frecuencia de $10 \mathrm{~Hz}$ y, en cada iteración, se convierten todas las medidas de los sensores a la magnitud física correspondiente y se guardan en el fichero estos valores, junto con el tiempo transcurrido desde que se inició la captura de datos, siempre que el programa se encuentre en el estado de captura. Además, también se registran los tiempos en los que el usuario ha pulsado el botón de registrar tiempo en el panel frontal. Esta funcionalidad permite cronometrar el tiempo que una persona necesita para completar una tarea.

\section{CASO DE ESTUDIO}

\subsection{Preparación del experimento}

Se realizó un ensayo con una persona sana para visualizar la fuerza calculada a partir de las medidas del sensor de fuerza y para comparar la inclinación obtenida de diferentes formas. Por un lado, se estimaron las inclinaciones de forma independiente a partir de las medidas de tres sensores integrados en la contera: el inclinómetro, el acelerómetro y el giróscopo. Por otro lado, se utilizó un sistema de visión para validar los valores de inclinación estimados mediante los tres sensores anteriores. La 
Figura 4 muestra un esquema de la disposición del recorrido del ensayo. Se puede observar que, para la validación de la inclinación anteroposterior, se colocó una cámara en perpendicular a la trayectoria de movimiento.

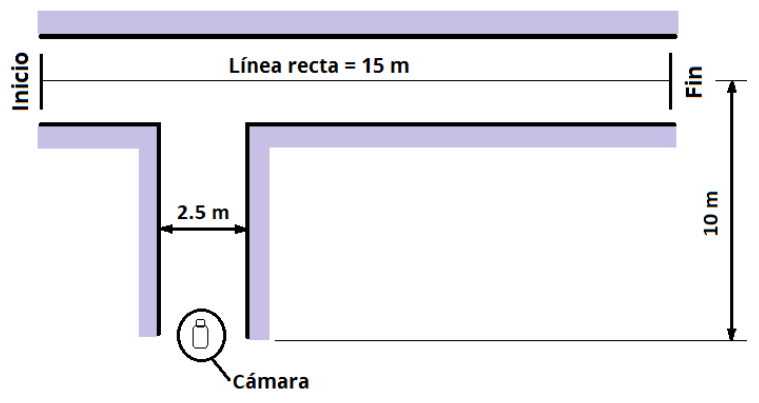

Figura 4: Esquema de la disposición del recorrido del ensayo.

En el ensayo, se le pidió a la participante que anduviera una distancia de 15 metros, siguiendo una línea recta y usando la muleta en su mano derecha, a su ritmo cómodo y normal. Además, se le pidió que apoyara el menor peso posible en su pie izquierdo. Antes de realizar el ejercicio, se le explicó que la muleta debe avanzar con el pie contralateral y se ajustó la altura de la muleta, de forma que el puño o apoyo de la muleta estuviera a la altura del extremo superior del fémur. El ensayo se realizó dos veces con la misma persona: una para validar la inclinación anteroposterior y otra para la inclinación lateromedial. En el último caso, la cámara se colocó en la misma dirección que la trayectoria. La voluntaria que participó en el ensayo firmó el consentimiento informado y el protocolo fue aprobado previamente por el comité de ética de la UPV/EHU con el código M10/2016/295MR1.

En el ensayo, se capturaron tanto los datos del sensor de fuerza para calcular la fuerza de apoyo mediante los parámetros obtenidos en la calibración, como los datos de la IMU y el inclinómetro, con el fin de estimar la inclinación anteroposterior de la muleta. Esta última es posible estimarla utilizando tanto el inclinómetro como los sensores de la IMU. Para obtener la estimación de la inclinación utilizando las medidas del inclinómetro, es suficiente aplicar la siguiente fórmula:

$$
\alpha=\arcsin \left(\frac{V_{\text {out }}-\text { Offset }}{2}\right)
$$

donde $V_{\text {out }}$ es la tensión medida por el inclinómetro y Offset es la tensión medida cuando la inclinación es cero.

Si por el contrario, se usan los datos de la IMU, existen otras dos posibilidades. Por un lado, inte- grar las medidas de velocidad angular en $\% / \mathrm{s}$ que nos ofrece este dispositivo. Por otro lado, usar los datos del acelerómetro integrado que, tratado con la siguiente expresión, permite una sensibilidad constante en todo el rango de medida [20]:

$$
\alpha=\arctan \frac{A_{x}}{\sqrt{A_{y}{ }^{2}+A_{z}^{2}}}
$$

donde $A_{x}, A_{y}$ y $A_{z}$ son las medidas filtradas del acelerómetro (en $\mathrm{m} / \mathrm{s}^{2}$ o en $\mathrm{g}$ ) en los ejes $\mathrm{X}, \mathrm{Y} \mathrm{y}$ $\mathrm{Z}$, respectivamente.

Con el objetivo de eliminar ruidos espurios, las medidas del inclinómetro, del acelerómetro y del giróscopo se han filtrado mediante un filtro de media móvil, con una ventana de $50 \mathrm{~ms}$.

Tras los ensayos, se extrajeron los fotogramas del video con el fin de medir el ángulo de la inclinación anteroposterior de la muleta, con una resolución de $1^{\circ}$. Esta señal obtenida mediante los fotogramas se usará como referencia real del movimiento de la muleta. La sincronización entre los datos extraídos de los fotogramas y las medidas de la myRIO se realizó suponiendo que el inicio del pico de fuerza se corresponde con el primer fotograma, en el que la contera está en contacto con el suelo.

\subsection{Resultados y análisis}

Los resultados del experimento anteriormente detallado se resumen el la Figura 5. En esta figura, se muestra la señal medida por el sensor de fuerza y las señales de la inclinación anteroposterior obtenidas mediante los sensores del prototipo (inclinómetro, acelerómetro y giróscopo) y a partir de los fotogramas. La señal roja se corresponde con la medida del sensor de fuerza (tras convertirla a $\mathrm{Kg}$ ). El resto de señales se refieren a la inclinación anteroposterior.

Se puede ver que, al inicio y al final de cada pico de fuerza, hay una barra vertical marrón. Estas barras marrones indican el comienzo y fin de las dos fases en cada ciclo de la marcha. Cada ciclo se puede dividir en dos fases [9]. Durante la fase de apoyo (stance), la punta de la muleta está en contacto con el suelo y el usuario está aplicando fuerza sobre esta. Durante la mayor parte de la fase de balanceo (swing), la punta de la muleta se mueve en el aire de atrás hacia adelante.

Dentro de la fase de apoyo, se pueden distinguir tres subfases [21]: la fase de contacto, la de medio apoyo y la de propulsión. Se observa que el valor de la fuerza en las fases de contacto y de propulsión es ligeramente superior a la de medio apoyo, debido a las fuerzas de impacto y 
propulsión ejercida por los músculos. Si se desea medir el porcentaje del peso corporal descargado sobre la muleta, se debe tomar la medida en la fase de medio apoyo, ya que es en este tramo donde se apoya el peso con fuerzas dinámicas despreciables [21].

Además, se puede observar que el valor mínimo de las medidas del sensor de fuerza es de más de $3 \mathrm{Kg}$. Esto se debe a que el comportamiento del sensor de fuerza no es lineal para fuerzas pequeñas y se calibró el sistema para el rango entre 10 y 60 Kg. Esta señal muestra que, a pesar de que se le pidiera que intentara cargar todo el peso de su cuerpo sobre la muleta, la participante solo cargó alrededor de $13 \mathrm{Kg}$, lo que corresponde al $14 \%$ de su peso corporal.

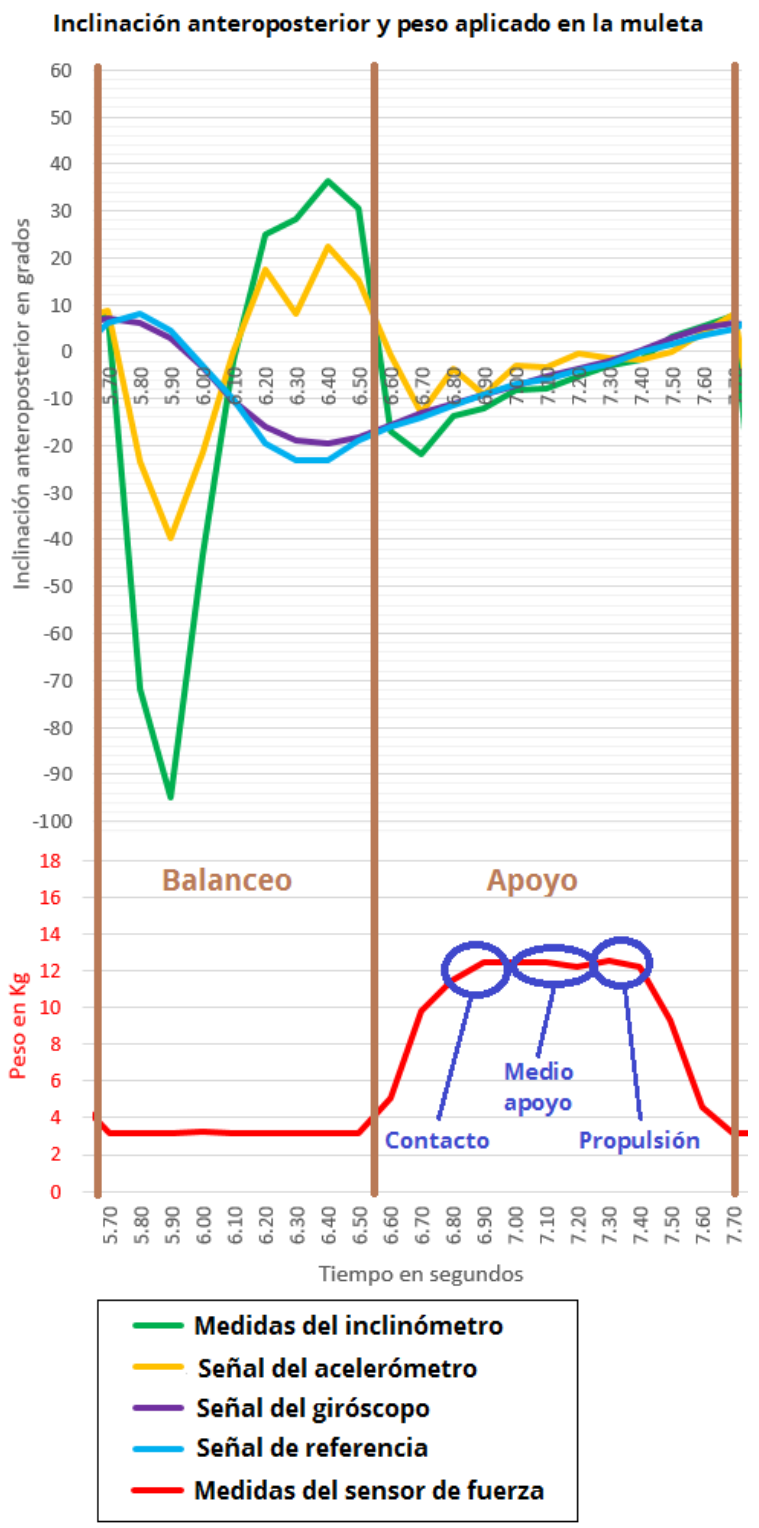

Figura 5: Medida del peso descargado y estimación de la inclinación anteroposterior de la muleta.
Por otro lado, valores negativos de la inclinación anteroposterior indican que la punta de la muleta está delante del cuerpo, mientras que valores positivos expresan que está detrás. La Figura 5 muestra que la mejor estimación de la inclinación anteroposterior en este caso se consigue integrando las medidas del giróscopo (morada), ya que es la señal que mejor se aproxima a la obtenida a partir de los fotogramas (azul clara). En el caso de la señal del giróscopo, el error de la raíz cuadrada de la media (RMSE) para el ciclo mostrado en la imagen es $1,83^{\circ}$.

Las señales del acelerómetro y del inclinómetro presentan pequeñas oscilaciones durante la fase de apoyo y grandes picos y oscilaciones durante la fase de balanceo. Esto sucede porque estos sensores son sensibles a los efectos dinámicos y las grandes aceleraciones provocan esos picos cuando la punta de la muleta se mueve en el aire o cuando impacta contra el suelo.

En la Figura 6, se muestran los resultados de la estimación de la inclinación lateromedial (con la misma leyenda que en la Figura 5). Valores más negativos indican que la contera está más lejos del cuerpo, en el plano frontal. La mejor estimación en este caso también se obtiene mediante el giróscopo y el error RMSE es $1,49^{\circ}$.

Pese a que en estos casos la señal del giróscopo se aproxima bastante bien a la de referencia y no se ve afectada por las aceleraciones dinámicas (las que no son causadas por la aceleración de la gravedad) ni por las perturbaciones magnéticas, el giróscopo tiene el inconveniente de la deriva en el tiempo [16]. El error de integración de la velocidad angular se va acumulando, lo que produce un offset considerable tras varios ciclos. Este es un aspecto en el que se trabajará en el futuro para garantizar una medida fiable a lo largo del tiempo.

\section{CONCLUSIONES}

Las escalas clínicas tradicionales para realizar un diagnóstico del estado funcional de los pacientes son subjetivas y de baja precisión. Sin embargo, los movimientos y fuerzas ejercidas por los pacientes se pueden monitorizar con mayor precisión mediante indicadores obtenidos a partir de las medidas de varios sensores. De este modo, se puede realizar una evaluación objetiva y cuantitativa de la mejora conseguida en el proceso de rehabilitación.

En este artículo, se ha presentado el diseño de una contera que tiene una serie de sensores integrados para monitorizar la inclinación de la muleta y la fuerza ejercida sobre su eje longitudinal, con el fin de estimar en un futuro diversos indicadores 


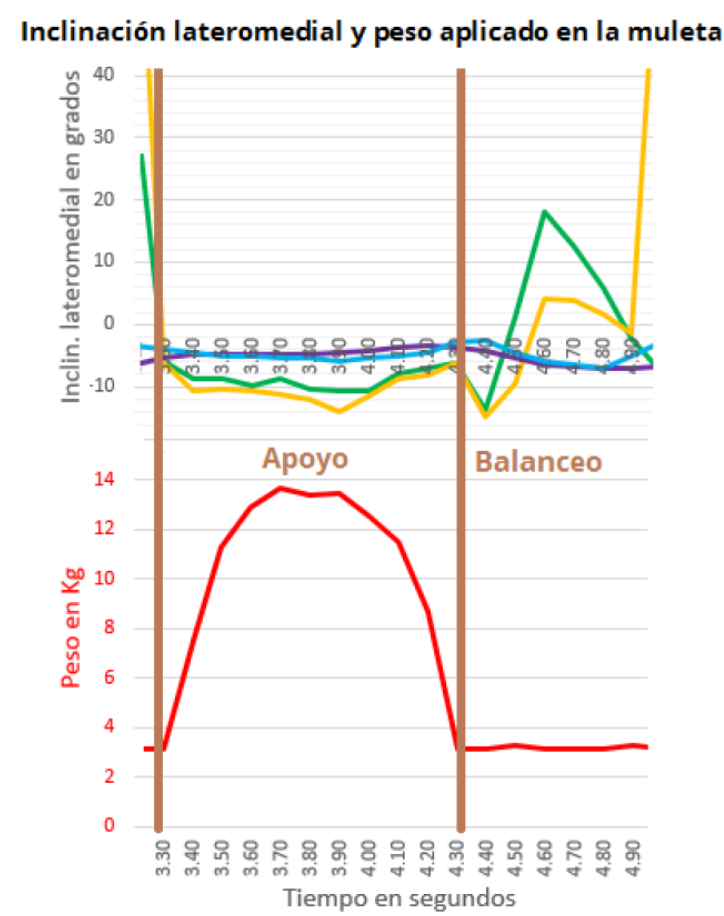

Figura 6: Medida del peso descargado y estimación de la inclinación lateromedial de la muleta.

que proporcionen un diagnóstico cuantitativo de la marcha. El prototipo proporciona una solución no invasiva, ligera y flexible, que permite monitorizar la marcha durante un tiempo prolongado en diversos entornos fuera del recinto hospitalario.

Tras resumir las especificaciones de diseño y proporcionar una descripción general de los componentes principales, se ha presentado el diseño mecánico y eléctrico, además de la arquitectura hardware y software. Para validar el prototipo y ver qué sensor es el más adecuado para estimar la inclinación, se ha analizado un caso de estudio. Se ha concluido que la estimación obtenida integrando las medidas del giróscopo es la que mejor se aproxima a la de referencia en este caso, con un error RMSE de $1,83^{\circ}$ para la inclinación anteroposterior y $1,49^{\circ}$ para la lateromedial.

\section{Agradecimientos}

Este trabajo ha recibido financiación del Gobierno Vasco mediante la beca PRE_2016_2_0236 y el proyecto IT914-16, de la Universidad del País Vasco (UPV/EHU) mediante el proyecto PPG17/56, de la Comisión Europea mediante la beca PN/TG1/UNSW/PhD/18/2017 y del Ministerio de Economía y Competitividad de España mediante el proyecto DPI2017-82694-R en MINECO FEDER.

\section{English summary}

Development of an instrumented crutch to measure inclination and discharged weight

\begin{abstract}
The traditional clinical scales used for the diagnosis of the functional state of patients with neurological disease are subjective and provide a low resolution. However, it is possible to include force and movement sensors in passive rehabilitation devices, in order to make an objective and quantitative diagnosis. This work presents the design of an instrumented crutch, which includes a force sensor, an inclinometer and an IMU, which consists of a triaxial accelerometer and gyroscope. In addition, it shows the values of the force and inclination, obtained in a case of study. The inclination estimated by the inclinometer and the IMU is validated using a camera.
\end{abstract}

Keywords: Crutch, cane, wearable sensors, monitoring, gait analysis.

\section{Referencias}

[1] Corbyn, Z. A Growing Global Burden. Nature 2014, 510, S3

[2] Lancini, M.; Serpelloni, M.; Pasinetti, S. Instrumented crutches to measure the internal forces acting on upper limbs in powered exoskeleton users. In Proceedings of 2015 6th IEEE International Workshop on Advances in Sensors and Interfaces. Gallipoli, Italy, 18-19 June, 2015. DOI. 10.1109/IWASI.2015.7184960

[3] Nordin, N.; Xie, S.Q.; Wünsche, B. Assessment of movement quality in robot-assisted upper limb rehabilitation after stroke: a review. Journal of Neuroengineering and Rehabilitation 2014, 11(137), 1-23, DOI. 10.1186/1743-0003-11-137

[4] Zhou, S.H.; Fong, J.; Crocher, V.; Tan, Y.; Oetomo, D.; Mareels, I. Learning control in robot-assisted rehabilitation of motor skills - a review. Journal of Control and Decision 2016, 3(1), 19-43, DOI. 10.1080/23307706.2015.1129295 
[5] Kim, C.M.; Eng, J.J. Magnitude and pattern of 3D kinematic and kinetic gait profiles in persons with stroke: relationship to walking speed. Gait \& Posture 2004, 20, 140-146, DOI. 10.1016/j.gaitpost.2003.07.002

[6] Tao, W; Liu, T; Zheng, R.; Feng, H. Gait analysis using wearable sensors. Sensors 2012, 12, 2255-2283, DOI. $10.3390 / \mathrm{s} 120202255$

[7] Bonato, P. Wearable Sensors/Systems and Their Impact on Biomedical Engineering. IEEE Engineering in Medicine and Biology Magazine 2003, 22:3, 18-20, DOI. 10.1109/MEMB.2003.1213622

[8] Park, S.; Jayaraman, S. Enhancing the quality of life through wearable technology. IEEE Engineering in Medicine and Biology Magazine. 2003, 22:3, 41-48, DOI. 10.1109/MEMB.2003.1213625

[9] Sardini, E.; Serpelloni, M.; Lancini, M. Wireless Instrumented Crutches for Force and Movement Measurements for Gait Monitoring. IEEE Transactions on Instrumentation and Measurement 2015, 64(12), 3369-3379, DOI. 10.1109/TIM.2015.2465751

[10] Chamorro-Moriana, G.; Sevillano, J.L.; Ridao-Fernández, C. A Compact Forearm Crutch Based on Force Sensors for Aided Gait: Reliability and Validity. Sensors 2016, 16(6), 1-15, DOI. 10.3390/s16060925

[11] Hassan, M.; Kadone, H.; Suzuki, K; Sankai, Y. Wearable Gait Measurement System with an Instrumented Cane for Exoskeleton Control. Sensors 2014, 14, 1705-1722, DOI. $10.3390 / \mathrm{s} 140101705$

[12] Mekki, F.; Borghetti, M.; Sardini, E.; Serpelloni, M. Wireless instrumented cane for walking monitoring in Parkinson patients. In Proceedings of 2017 IEEE International Symposium on Medical Measurements and Applications (MeMeA). Rochester, MN, USA, 7-10 May, 2017. DOI. 10.1109/MeMeA.2017.7985912

[13] Lemay, M.; Bertschi, M.; Sola, J.; Renevey, P.; Parak, J.; Kornhonen, I. Application of Optical Heart Rate Monitoring. En Wearable Sensors: Fundamentals, Implementation and Applications; Sazonov, E., Neuman, M.R., Eds.; Elsevier: USA, 2014; Chapter 2.3, pp. 105-129, ISBN 978-0-12-418662-0

[14] Motion Workshop. Shadow Full Body Motion Capture. 2013. https://vimeo.com/ 57986224 (Accedido el 12/06/2018)
[15] Wolfe, J. MPC Puts New XSens Motion Capture System to Work. Animation World Network, 2015. https://www. awn.com/news/ mpc-puts-new-xsens-motion-capture-/ system-work (Accedido el 12/06/2018)

[16] Tsuda, N.; Hayashi, A.; Tounai, M.; Akutagawa, S. Visualization System of Crutch Walking based on Internal Sensors. In Proceedings of 2010 IEEE/ASME International Conference on Advanced Intelligence Mechatronics. Montréal, Canada, 6-9 July, 2010.

[17] Chamorro-Moriana, G.; Ridao-Fernández, C.; Ojeda, J.; Benítez-Lugo, M.; Sevillano, J.L. Reliability and Validity Study of the Chamorro Assisted Gait Scale for People with Sprained Ankles, Walking with Forearm Crutches. PLoS One 2016, 11(5), 1-12, DOI. 10.1371/journal.pone. 0155225

[18] Van Laarhoven, S.N.; Lipperts, M.; Bolink, S.A.A.N.; Senden, R.; Heyligers, I.C.; Grimm, B. Validation of a novel activity monitor in impaired, slow-walking, crutchsupported patients. Annals of Physical and Rehabilitation Medicine 2016, 59, 308-313, DOI. 10.1016/j.rehab.2016.05.006

[19] Mathie, M.J.; Coster, A.C.F.; Lovell, N.H.; Celler, B.G.; Lord, S.R.; Tiedemann, A. A pilot study of long-term monitoring of human movements in the home using accelerometry. Journal of Telemedicine and Telecare 2004, 10, 144-151, DOI. 10.1258/135763304323070788

[20] Tuck, K. Tilt Sensing Using Linear Accelerometers. Available online: https://www.mh-hannover.de/fileadmin/ zentrale_einrichtungen/zentrale_ forschungswerkst/downloads/AN3461.pdf (accedido el 18 de junio de 2018)

[21] Merrett, G.V.; Ettabib, M.A.; Peters, C.; Hallett, G.; White, N.M. Augmenting forearm crutches with wireless sensors for lower limb rehabilitation. Measurement Science and Technology 2010, 21, 1-10, DOI. 10.1088/0957-0233/21/12/124008

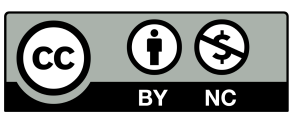

(c) 2018 by the authors. Submitted for possible open access publication under the terms and conditions of the Creative Commons Attribution CC-BY-NC 3.0 license (http://creativecommons.org/licenses/by-nc/3.0/). 